\title{
PERMANENT CONFIGURATIONS IN THE PROBLEM OF FIVE BODIES*
}

\author{
BY \\ W. L. WILLIAMS
}

1. Introduction. In 1772 Lagrange found that if three bodies of arbitrary masses are placed at the vertices of an equilateral triangle and given the proper initial velocities, they will continue to be at the vertices of an equilateral triangle which may change in size, but not in shape. He also showed that if the three bodies are placed in a straight line and started off suitably, they will remain in a straight line, and the ratios of the mutual distances will be constant. More recently Moulton $\dagger$ has extended the straight line configuration to $n$ bodies, and some particular instances of other configurations have been given by Hoppe, $\ddagger$ Andoyer, $\S$ and Longley. $\|$

A permanent configuration is a configuration of $n$ bodies which has the property that the ratio of distances between corresponding bodies is constant. In other words, the figure may change in size, but not in shape. The object of this paper is to determine necessary and sufficient conditions for any plane configuration of five bodies, other than a straight line configuration, to be permanent and to give a detailed analysis of the various types of such configurations, both convex and concave, that can be formed by rearranging the bodies to give pentagons of different shapes. This work may be regarded as an extension of that of MacMillan and Bartky $\uparrow$ in which the problem of four bodies is treated.

2. The equations of motion. Let $m_{1}, m_{2}, \cdots, m_{5}$ with coordinates $\left(x_{1}, y_{1}\right)$, $\left(x_{2}, y_{2}\right), \cdots,\left(x_{5}, y_{5}\right)$, respectively, represent the masses of a plane system of five bodies referred to a set of rectangular axes, with origin at their common center of mass, which rotates with uniform angular velocity $\omega$. If we assume that the bodies attract each other according to the Newtonian law and move in circles around the origin with uniform angular velocity $\omega$, the differential

* Presented to the Society, September 6, 1938; received by the editors May 20, 1937.

$\dagger$ Periodic Orbits, Carnegie Institution, 1920, p. 285.

† Erweiterung der bekannten Speciallösung des Dreikörperproblems, Archiv der Mathematik und Physik, vol. 64, p. 218.

$\S$ Sur l'équilibre relatif de $n$ corps, Bulletin Astronomique, vol. 23 (1906), p. 50.

II Some particular solutions in the problem of $n$ bodies, Bulletin of the American Mathematical Society, vol. 13 (1906-1907), p. 324.

T Permanent configurations in the problem of four bodies, these Transactions, vol. 34 (1932), pp. 838-875. 
equations of motion reduce to

$$
\begin{aligned}
\omega^{2} x_{i} & =\sum_{j=1}^{5} \frac{m_{j}\left(x_{i}-x_{j}\right)}{r_{i, j}^{3}}, \\
\omega^{2} y_{i} & =\sum_{j=1}^{5} \frac{m_{j}\left(y_{i}-y_{j}\right)}{r_{i, j}^{3}},
\end{aligned}
$$

where $r_{i, j}$ is the distance between the masses $m_{i}$ and $m_{j}$.

It is to be observed that the equations

$$
\sum_{j=1}^{5} m_{i} x_{i}=0, \quad \sum_{i=1}^{5} m_{i} y_{i}=0,
$$

which express the fact that the origin of coordinates is at the center of mass, follow directly from (1).

Define a constant $r_{0}$ by

$$
\omega^{2}=\sum_{i=1}^{5} m_{i} / r_{0}^{3} .
$$

The constant $r_{0}$ is to replace $\omega$ and will be used later in defining the regions for positive masses.

The equations (1), as a consequence of (2) and (3), may now be written in the equivalent form

$$
\begin{aligned}
& \sum_{j=1}^{5} S_{i, j}\left(x_{i}-x_{j}\right) m_{j}=0, \\
& \sum_{j=1}^{5} S_{i, j}\left(y_{i}-y_{j}\right) m_{i}=0,
\end{aligned}
$$

where

$$
S_{i, j}=S_{j, i}=R_{i, j}-R_{0}, \quad R_{i, j}=R_{i, i}=1 / r_{i, j}^{3}, \quad R_{0}=1 / r_{0}^{3} .
$$

If in (4) $m_{i}$ and $m_{i+1},(i=1,2, \cdots, 5)$, are eliminated, respectively, from the $(i+1)$ th and $i$ th equations in $x$ and the corresponding equations in $y$, and if we denote twice the area of the triangle whose vertices have as coordinates any three of the points $\left(x_{i}, y_{i}\right),(i=1,2, \cdots, 5)$, by $D$ with a pair of numbers (from 1 to 5 ) as subscripts which do not occur as subscripts in the coordinates of the vertices of the triangle, we have

$$
\begin{aligned}
& S_{i, i+3} D_{i+1, i+2} m_{i}+S_{i+1, i+3} D_{i, i+2} m_{i+1}+S_{i+2, i+3} D_{i, i+1} m_{i+2}=0, \\
& S_{i, i+4} D_{i+1, i+2} m_{i}+S_{i+1, i+4} D_{i, i+2} m_{i+1}+S_{i+2, i+4} D_{i, i+1} m_{i+2}=0,
\end{aligned}
$$




$$
\begin{aligned}
& m_{i} /\left(S_{i+1, i+3} S_{i+2, i+4}-S_{i+1, i+4} S_{i+2, i+3}\right) D_{i, i+2} D_{i, i+1} \\
& \quad=m_{i+1} /\left(S_{i+2, i+3} S_{i, i+4}-S_{i+2, i+4} S_{i, i+3}\right) D_{i+1, i+2} D_{i, i+1} \\
& =m_{i+2} /\left(S_{i, i+3} S_{i+1, i+4}-S_{i, i+4} S_{i+1, i+3}\right) D_{i+1, i+2} D_{i, i+2}, \\
& \quad i=1,2, \cdots, 5,
\end{aligned}
$$

a system of equations, equivalent to (4), in which the variables $x$ and $y$ do not occur explicitly. It is to be understood here as well as hereafter that the subscripts $6,7,8$, and 9 are to be replaced by $1,2,3$, and 4 , respectively.

For each value of $i$ in equations (6), two independent relations may be formed, each expressing a mass ratio as a function of the $D$ 's and $S$ 's. Among these ten mass ratios six distinct equalities exist; and when (6) is substituted in them we obtain, after some reduction,

$$
\frac{S_{i, i+3} S_{i+1, i+2}-S_{i, i+2} S_{i+1, i+3}}{S_{i, i+2} S_{i+1, i+3}-S_{i, i+1} S_{i+2, i+3}}=\lambda_{i+4}, \quad i=1,2, \cdots, 5,
$$

where

$$
\lambda_{i+4}=\frac{D_{i+2, i+3} D_{i, i+1}}{D_{i+1, i+2} D_{i, i+3}}
$$

It is to be observed that the subscript on $\lambda$ corresponds to the missing subscript in the left-hand members of (7).

One may inquire whether the denominators in (6) may not vanish under certain conditions. That this is impossible may be seen by considering the several possible cases.

It should be stated first that in the five-body problem the masses are all different from zero. The form of equations (1) shows that this hypothesis has been made. Suppose, now, that no three of the bodies are in the same straight line (so that no $D$ is zero), and let one of the expressions in $S$, say $S_{24} S_{35}-S_{25} S_{34}$, vanish. It follows at once from (6) that

$$
S_{i, i+1} S_{i+2, i+4}=S_{i, i+2} S_{i+1, i+4}=S_{i, i+4} S_{i+1, i+2}, \quad i=1,2, \cdots, 5 .
$$

That is, if one denominator in (6) vanishes, all must vanish. But it is impossible to satisfy all of the equations (A) simultaneously, for if it were, then equations (5) would show that a permanent configuration of five bodies is obtained in which the five masses are arbitrary. Let two of these masses approach zero. The result, in so far as finite masses are concerned, is a permanant configuration of three bodies with arbitrary masses. The only such figure is an equilateral triangle. Hence, if the equations (A) are satisfied by a pentagon configuration, any three vertices of this pentagon must form an equilateral triangle. This is geometrically impossible. 
Consider next the case where three or more of the bodies are in the same straight line. The case in which they are all in a line has been treated by Moulton, and the method of this paper is not applicable. Outside of Moulton's case there are only two five-body configurations in which some of the denominators in (6) vanish; namely, the square and the rhombus with one mass at each vertex and the fifth mass at the center. These two cases are treated in Examples 2 and 3 on pages 578 and 579, respectively. Hence no omission of cases arises through regarding the denominators in (6) as non-zero.

3 . The relations among the triangular area ratios. We assume that in general no three of the points $\left(x_{i}, y_{i}\right),(i=1,2, \cdots, 5)$, lie in the same straight line. Hence they uniquely determine a conic, and this conic may be projected into a circle. In this process of projection the triangular area ratios remain unchanged; that is, the $\lambda$ 's defined in (8) are invariant under projection. If we make use of this property, we find that when the $D$ 's are eliminated from (8), the $\lambda$ 's satisfy a system of equations of the form

$$
\lambda_{i}-\lambda_{i+2} \lambda_{i+3}-\lambda_{i} \lambda_{i+2} \lambda_{i+3}=0,
$$$$
i=1,2, \cdots, 5 \text {. }
$$

4. A geometric property of the $\lambda$ 's. Let us consider, as typical of the triangular area ratios, the first one, which by (8) is

$$
\lambda_{5}=D_{12} D_{34} / D_{14} D_{23} \text {. }
$$

If the points $1,2,3$, and 4 are assigned coordinates and $\lambda_{5}$ is assigned a value, then this equation represents a conic whose variables are the coordinates of the point 5 , the nature of the conic depending upon the value given to $\lambda_{5}$. The conic obviously passes through the points $1,2,3$, and 4 . It will be useful later to know under what condition the conic will degenerate into a pair of straight lines.

Let the points $1,2,3$, and 4 have, respectively, the coordinates $(0,0)$, $\left(x_{2}, 0\right),\left(x_{3}, y_{3}\right)$, and $\left(x_{4}, y_{4}\right)$, let the coordinates of the point 5 be $(x, y)$, and omit the subscript on $\lambda_{5}$. Then if the equation of the conic is written out and the condition for a conic to degenerate into a pair of straight lines is imposed, it is found that

$$
\lambda(\lambda+1)=0 .
$$

Hence we have the following theorem:

THEOREM 4.1. If, in any one of the triangular area ratios, the point indicated by the subscript on the associated $\lambda$ is allowed to vary while the other four points are fixed, the variable point will move in a conic which passes through the four fixed points and will degenerate into a pair of straight lines if, and only if, $\lambda$ has either the value 0 or -1 . 
5. The necessary condition. In order that the problem may admit a solution other than the straight line solution, it is necessary that equations (7) be satisfied. However, if any three of these equations are satisfied, the remaining two are also satisfied, since any set of three of them implies the other two. For instance, suppose we write down the following three of these equations:

$$
\begin{aligned}
& S_{14} S_{23}-S_{13} S_{24}\left(1+\lambda_{5}\right)+S_{12} S_{34} \lambda_{5}=0 \\
& S_{15} S_{24}-S_{14} S_{25}\left(1+\lambda_{3}\right)+S_{12} S_{45} \lambda_{3}=0, \\
& S_{25} S_{34}-S_{24} S_{35}\left(1+\lambda_{1}\right)+S_{23} S_{45} \lambda_{1}=0,
\end{aligned}
$$

which correspond, respectively, to the values 1,4 , and 2 of $i$, and write down the one corresponding to $i=5$, namely,

$$
S_{12} S_{35}-S_{13} S_{25}\left(1+\lambda_{4}\right)+S_{15} S_{23} \lambda_{4}=0 .
$$

To show that this equation is implied by equations (10) multiply the second equation of (10) by $\lambda_{1} S_{23}$ and the third one by $\lambda_{3} S_{12}$ and subtract, thus eliminating $S_{45}$. Multiply this result throughout by $\lambda_{5}$ and the first equation of (10) by $\lambda_{3}$. Then, using the relation $\lambda_{3}=\lambda_{1} \lambda_{5}+\lambda_{1} \lambda_{3} \lambda_{5}$ from (9) and subtracting, we have

$$
S_{13} S_{25}\left(1+\lambda_{5}\right)-S_{12} S_{35}\left(1+\lambda_{1}\right) \lambda_{5}-S_{15} S_{23}\left(1-\lambda_{1} \lambda_{5}\right)=0 .
$$

In order to see that this equation is identical with (10a), one only has to eliminate $\lambda_{2}$ from the second and fourth of the $\lambda$ equations (9) obtaining $\lambda_{4}=\left(1-\lambda_{1} \lambda_{5}\right) /\left(1+\lambda_{1}\right) \lambda_{5}$, and substitute this expression in (10a).

6. Convex and concave pentagons. Suppose that pegs are placed at each of the five masses and a string is drawn tautly around them. Two cases are distinguishable:

Case 1. The string touches all five pegs, and

(a) no three pegs are in a straight line;

(b) three pegs are in a straight line, but not four;

(c) four pegs are in a straight line, but not five;

(d) five pegs are in a straight line.

Case 2 (a). The string touches only four pegs, the fifth one being inside the quadrilateral formed by the other four.

Case 2 (b). The string touches only three pegs, the other two being inside the triangle formed by the first three.

If the conditions of Case 1 (a), 1 (b), or 1 (c) are satisfied, the pentagon will be called convex. Case 1 (d) is the straight line configuration with which we are not concerned. If the conditions of Case 2 (a) or Case 2 (b) are satisfied, the pentagon will be called concave. 
In all cases we shall regard the masses as arranged in the counter-clockwise order $m_{1}, m_{2}, m_{3}, m_{4}$, and $m_{5}$.

From equations (6) we may write

$$
\begin{aligned}
& m_{1}=\frac{D_{12}}{D_{23}} \frac{\left(R_{24}-R_{0}\right)\left(R_{35}-R_{0}\right)-\left(R_{34}-R_{0}\right)\left(R_{25}-R_{0}\right)}{\left(R_{14}-R_{0}\right)\left(R_{25}-R_{0}\right)-\left(R_{24}-R_{0}\right)\left(R_{15}-R_{0}\right)} m_{3}, \\
& m_{2}=\frac{D_{12}}{D_{13}} \frac{\left(R_{34}-R_{0}\right)\left(R_{15}-R_{0}\right)-\left(R_{14}-R_{0}\right)\left(R_{35}-R_{0}\right)}{\left(R_{14}-R_{0}\right)\left(R_{25}-R_{0}\right)-\left(R_{15}-R_{0}\right)\left(R_{24}-R_{0}\right)} m_{3} \text {, } \\
& m_{2}=\frac{D_{24}}{D_{34}} \frac{\left(R_{13}-R_{0}\right)\left(R_{45}-R_{0}\right)-\left(R_{14}-R_{0}\right)\left(R_{35}-R_{0}\right)}{\left(R_{14}-R_{0}\right)\left(R_{25}-R_{0}\right)-\left(R_{12}-R_{0}\right)\left(R_{45}-R_{0}\right)} m_{3} \text {, } \\
& m_{4}=\frac{D_{24}}{D_{23}} \frac{\left(R_{12}-R_{0}\right)\left(R_{35}-R_{0}\right)-\left(R_{13}-R_{0}\right)\left(R_{25}-R_{0}\right)}{\left(R_{14}-R_{0}\right)\left(R_{25}-R_{0}\right)-\left(R_{12}-R_{0}\right)\left(R_{45}-R_{0}\right)} m_{3} \text {, } \\
& m_{4}=\frac{D_{45}}{D_{35}} \frac{\left(R_{13}-R_{0}\right)\left(R_{25}-R_{0}\right)-\left(R_{23}-R_{0}\right)\left(R_{15}-R_{0}\right)}{\left(R_{24}-R_{0}\right)\left(R_{15}-R_{0}\right)-\left(R_{14}-R_{0}\right)\left(R_{25}-R_{0}\right)} m_{3} \text {, } \\
& m_{5}=\frac{D_{45}}{D_{34}} \frac{\left(R_{14}-R_{0}\right)\left(R_{23}-R_{0}\right)-\left(R_{13}-R_{0}\right)\left(R_{24}-R_{0}\right)}{\left(R_{24}-R_{0}\right)\left(R_{15}-R_{0}\right)-\left(R_{14}-R_{0}\right)\left(R_{25}-R_{0}\right)} m_{3}, \\
& m_{1}=\frac{D_{14}}{D_{45}} \frac{\left(R_{24}-R_{0}\right)\left(R_{35}-R_{0}\right)-\left(R_{34}-R_{0}\right)\left(R_{25}-R_{0}\right)}{\left(R_{12}-R_{0}\right)\left(R_{34}-R_{0}\right)-\left(R_{13}-R_{0}\right)\left(R_{24}-R_{0}\right)} m_{5} \text {, } \\
& m_{4}=\frac{D_{14}}{D_{15}} \frac{\left(R_{13}-R_{0}\right)\left(R_{25}-R_{0}\right)-\left(R_{12}-R_{0}\right)\left(R_{35}-R_{0}\right)}{\left(R_{12}-R_{0}\right)\left(R_{34}-R_{0}\right)-\left(R_{13}-R_{0}\right)\left(R_{24}-R_{0}\right)} m_{5} \text {, } \\
& m_{1}=\frac{D_{12}}{D_{25}} \frac{\left(R_{23}-R_{0}\right)\left(R_{45}-R_{0}\right)-\left(R_{24}-R_{0}\right)\left(R_{35}-R_{0}\right)}{\left(R_{13}-R_{0}\right)\left(R_{24}-R_{0}\right)-\left(R_{14}-R_{0}\right)\left(R_{23}-R_{0}\right)} m_{5} \text {, } \\
& m_{2}=\frac{D_{12}}{D_{15}} \frac{\left(R_{14}-R_{0}\right)\left(R_{35}-R_{0}\right)-\left(R_{13}-R_{0}\right)\left(R_{45}-R_{0}\right)}{\left(R_{13}-R_{0}\right)\left(R_{24}-R_{0}\right)-\left(R_{14}-R_{0}\right)\left(R_{23}-R_{0}\right)} m_{5} \text {. }
\end{aligned}
$$

For a convex pentagon, the ten triangular area ratios in these equations satisfy the inequalities

$$
\begin{aligned}
& D_{12} / D_{23}>0, D_{12} / D_{13}>0, D_{24} / D_{34}>0, D_{24} / D_{23}>0, \\
& D_{45} / D_{35}>0, D_{45} / D_{34}>0, D_{14} / D_{45}>0, D_{14} / D_{15}>0 \text {, } \\
& D_{12} / D_{25}>0, D_{12} / D_{15}>0 \text {. }
\end{aligned}
$$

For the general concave pentagon of the type described in Case 2 (a), there are four different positions that the fifth mass may occupy within the quadrilateral formed by the other four masses. These positions are shown in Figs. $1 \mathrm{a}, 1 \mathrm{~b}, 1 \mathrm{c}$, and $1 \mathrm{~d}$. The following table gives the signs of the triangular area ratios for each of these four cases: 


\begin{tabular}{|c|c|c|c|c|c|c|c|c|c|c|}
\hline Figure & $\frac{D_{12}}{D_{23}}$ & $\frac{D_{12}}{D_{13}}$ & $\frac{D_{24}}{D_{34}}$ & $\frac{D_{24}}{D_{23}}$ & $\frac{D_{45}}{D_{35}}$ & $\frac{D_{45}}{D_{34}}$ & $\frac{D_{14}}{D_{45}}$ & $\frac{D_{14}}{D_{15}}$ & $\frac{D_{12}}{D_{25}}$ & $\frac{D_{12}}{D_{15}}$ \\
\hline $1 \mathrm{a}$ & - & + & + & + & + & + & + & + & - & - \\
\hline $1 \mathrm{~b}$ & - & + & + & + & + & + & + & + & + & - \\
\hline $1 \mathrm{c}$ & - & - & + & + & + & + & + & + & + & - \\
\hline $1 \mathrm{~d}$ & - & - & + & + & + & + & + & + & - & - \\
\hline
\end{tabular}

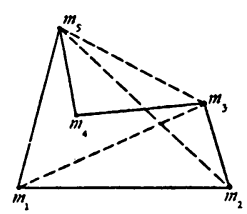

Fig. 1a

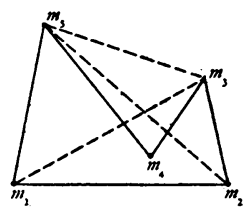

FIG. 1b

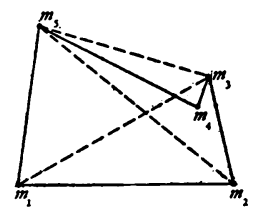

FIg. 1c

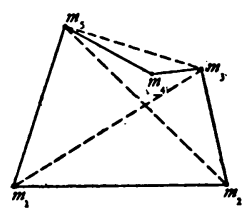

Frg. 1d

7. Admissible convex pentagons. An admissible pentagon is one for which there exist positive masses which, when placed at its vertices and started off suitably, will give a permanent configuration.

In any solution of the problem, one sees from (10) that the following equations

$$
\begin{aligned}
& \frac{\left(R_{14}-R_{0}\right)\left(R_{23}-R_{0}\right)-\left(R_{13}-R_{0}\right)\left(R_{24}-R_{0}\right)}{\left(R_{13}-R_{0}\right)\left(R_{24}-R_{0}\right)-\left(R_{12}-R_{0}\right)\left(R_{34}-R_{0}\right)}=\frac{D_{12} D_{34}}{D_{14} D_{23}}, \\
& \frac{\left(R_{24}-R_{0}\right)\left(R_{15}-R_{0}\right)-\left(R_{14}-R_{0}\right)\left(R_{25}-R_{0}\right)}{\left(R_{14}-R_{0}\right)\left(R_{25}-R_{0}\right)-\left(R_{12}-R_{0}\right)\left(R_{45}-R_{0}\right)}=\frac{D_{12} D_{45}}{D_{24} D_{15}}, \\
& \frac{\left(R_{34}-R_{0}\right)\left(R_{25}-R_{0}\right)-\left(R_{24}-R_{0}\right)\left(R_{35}-R_{0}\right)}{\left(R_{24}-R_{0}\right)\left(R_{35}-R_{0}\right)-\left(R_{23}-R_{0}\right)\left(R_{45}-R_{0}\right)}=\frac{D_{23} D_{45}}{D_{34} D_{25}},
\end{aligned}
$$

must be satisfied, and in addition, for any solution, equations (12) must yield positive masses.

Since for convex pentagons the inequalities (13) must hold, and since the masses are necessarily positive, it follows that the numerator and denominator involving the $R$ 's in each of the equations (12) must be of like sign. If we let the numerator and denominator of the first equation be positive, the signs of the numerator and denominator in each of the other equations are determined, and we find that 


$$
\begin{aligned}
\left(R_{i, i+1}-R_{0}\right)\left(R_{i+2, i+3}-\right. & \left.R_{0}\right)>\left(R_{i, i+2}-R_{0}\right)\left(R_{i+1, i+3}-R_{0}\right) \\
& >\left(R_{i, i+3}-R_{0}\right)\left(R_{i+1, i+2}-R_{0}\right), \quad i=1, \cdots, 5 .
\end{aligned}
$$

We can choose three of the points arbitrarily. Let these be the first three. We then have given $r_{12}, r_{23}$, and $r_{13}$ and can therefore choose their order relation. Let this relation be

$$
r_{12}, r_{23} \leqq r_{0} \leqq r_{13} .
$$

Draw $r_{12}$ as in Fig. 2, and let $m_{1}$ and $m_{2}$ be at its extremities. With $m_{1}$ and $m_{2}$ as centers draw semicircles with radius $r_{0}$, intersecting at $O$. With $O$ as center describe circular arc $a_{1} b_{1} c_{4} b_{2} a_{2}$ with radius $r_{0}$. Then with $m_{3}$ in the region $O a_{2} b_{2}$, $m_{4}$ in the region bounded by circular arcs $O b_{1}, O b_{2}$, and $b_{1} c_{4} b_{2}$, and $m_{5}$ in the region $O a_{1} b_{1}$, the inequalities (15) are all satisfied, subject to the condition

$$
r_{12}, r_{23}, r_{34}, r_{15}, r_{45} \leqq r_{0} \leqq r_{13}, r_{14}, r_{24}, r_{25}, r_{35}
$$

which assures that the masses in the regions specified are all positive. The proof that with the masses in these regions equations (14) are satisfied is too long to write down here. Only the method of procedure will be indicated.

In Fig. 2 take $m_{3}$ in its region as shown or on the boundary of this region, and with this point as center and with $r_{0}$ as radius describe circular $\operatorname{arc} c_{1} c_{2} c_{3} c_{4}$, intersecting arc $O a_{1}$ at $c_{2}$, arc $O b_{1}$ at $c_{3}$, and arc $b_{1} b_{2}$ at $c_{4}$. On account of the inequalities (16), the mass $m_{4}$ is further restricted to the region $O c_{3} c_{4} b_{2}$. Let this region be covered with an infinity of arcs, all passing through the point $O$ and intersecting the boundary $\operatorname{arc} c_{3} c_{4}$. It can be shown that on each of these arcs there is one and but one point at which the first of equations (14) is satisfied. If these points are joined by a curve, we have the curve on which point $m_{4}$ moves as $m_{3}$ varies its position in the region $O a_{2} b_{2}$. Imagine $m_{4}$ on this curve, and with it as center and $r_{0}$ as radius describe circular arc $a_{3} b_{3}$ intersecting $O b_{1}$ at $b_{3}$ and $O a_{1}$ at some point $a_{3}$ below $c_{2}$. The remaining two equations of (14), then, determine two curves which pass through $m_{4}$ and intersect in the region $c_{2} c_{3} b_{3} a_{3}$, giving the position of $m_{5}$. As the point $m_{4}$ varies its position on its curve, $m_{5}$ moves on some curve in the region $c_{2} c_{3} b_{3} a_{3}$. These results lead to the following theorem:

THEOREM 7.1. For every point $m_{3}$ in the region $\mathrm{Ob}_{2} a_{2}$, or on the boundary of his region, there exists a single infinity of points $m_{4}$ in the region $\mathrm{Oc}_{3} c_{4} b_{2}$ and a single infinity of points $m_{5}$ in the region $O a_{1} b_{1}$, which together with the points $m_{1}$ and $m_{2}$ form an admissible convex pentagon.

8. Limitations on the interior angles. In Fig. 2 let the angles of the pentagon at its five vertices be denoted by the masses at these points. For example, angle $m_{2} m_{1} m_{5}$ will be denoted by $m_{1}$, and angle $m_{1} m_{2} m_{3}$ by $m_{2}$. 
It is obvious that angle $m_{1}$ is equal to or greater than angle $m_{2} m_{1} O$ and that this latter angle obtains its least value when $r_{12}=r_{0}$. This value is seen to be $60^{\circ}$ from Fig. 3, which is the same as Fig. 2 except that it is drawn with $r_{0}=r_{12}$. Therefore angle $m_{1}$ is equal to or greater than $60^{\circ}$, and the same is clearly true for angle $m_{2}$.

Since $r_{12}=r_{0}$, the first of equations (14) shows that the point $m_{4}$ moves on some curve through the point $O$, such as curve $n$, Fig. 3 . We see, however, that the smallest value of angle $m_{3}$ is obtained when point $m_{3}$ is at $b_{2}$ and point $m_{4}$ is at $O$, in which case it is $60^{\circ}$. By symmetry it follows that the smallest value of angle $m_{5}$ is also $60^{\circ}$ and is obtained when point $m_{4}$ is at $O$ and point $m_{5}$ is at $b_{1}$.

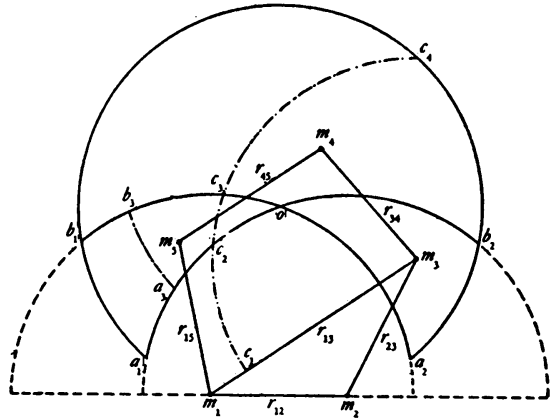

FIG. 2

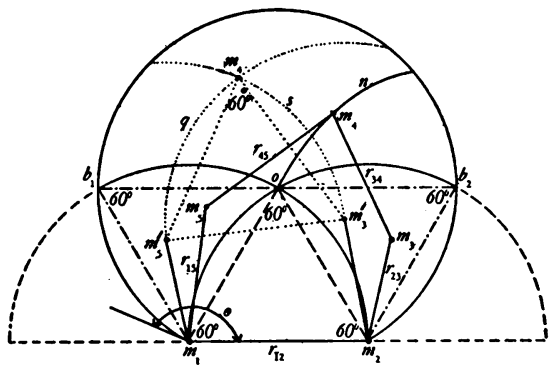

FIG. 3

Consider now angle $m_{4}$. In Fig. 3, let points $m_{3}$ and $m_{5}$ be at $m_{3}^{\prime}$ and $m_{6}^{\prime}$, respectively, so that $r_{35}$ equals $r_{0}$, its least value. With these two points as centers describe $\operatorname{arcs} q$ and $s$, respectively, each with radius $r_{0}=r_{12}=r_{35}$. From the inequality (16) we see that the point $m_{4}$ cannot lie outside of the region bounded by the $\operatorname{arcs} q, s$ and the arcs of the circles drawn with the points $m_{1}$ and $m_{2}$ as centers. It is clear then that angle $m_{4}$ is smallest when point $m_{4}$ lies at the intersection of $\operatorname{arcs} q$ and $s$, and that it is then $60^{\circ}$.

Hence no interior angle of the convex pentagon can be less than $60^{\circ}$.

The maximum value of the angle $m_{1}$ is also reached when $r_{12}=r_{0}$, as can be seen from Figs. 2 and 3, and is that angle $\theta$, Fig. 3, which the circle with $O$ as center makes with the line $r_{12}$.

In order to find $\theta$ let the line through the points $m_{1}$ and $m_{2}$ be the $x$ axis, and let these two points be symmetric with respect to the origin, so that the point $O$ will be on the $y$ axis. Then if the equation of the circle with $O$ as center and $r_{0}$ as radius is formed, it follows easily that the angle $\theta$ which it makes with the line $r_{12}$ is $150^{\circ}$. 
It is obvious from Fig. 3 that angle $m_{2}$ also has this angle for its maximum value; and this is true for angles $m_{3}, m_{4}$, and $m_{5}$ as well, as can be shown by going through the same procedure for these angles as for angle $m_{1}$.

THEOREM 8.1. Each of the interior angles of an admissible convex pentagon lies between $60^{\circ}$ and $150^{\circ}$.

9. Admissible concave pentagons. We shall consider the general case where one of the points $m_{i}$ is within the quadrilateral formed by the other four. Let the vertices of the quadrilateral be $m_{1}, m_{2}, m_{3}$, and $m_{5}$, and let the interior point be $m_{4}$ whose position is that shown in Fig. 1d.

For concave pentagons of the type under consideration, the signs indicated in the last row in the table on page 569 must obtain, and since the masses are positive, the $R$ 's in equations (12) must satisfy the relations

$$
\begin{aligned}
& \left(R_{12}-R_{0}\right)\left(R_{45}-R_{0}\right)>\left(R_{14}-R_{0}\right)\left(R_{25}-R_{0}\right)<\left(R_{24}-R_{0}\right)\left(R_{15}-R_{0}\right), \\
& \left(R_{34}-R_{0}\right)\left(R_{15}-R_{0}\right)>\left(R_{14}-R_{0}\right)\left(R_{35}-R_{0}\right)>\left(R_{13}-R_{0}\right)\left(R_{45}-R_{0}\right), \\
& \left(R_{34}-R_{0}\right)\left(R_{25}-R_{0}\right)<\left(R_{24}-R_{0}\right)\left(R_{35}-R_{0}\right)>\left(R_{23}-R_{0}\right)\left(R_{45}-R_{0}\right), \\
& \left(R_{23}-R_{0}\right)\left(R_{15}-R_{0}\right)<\left(R_{13}-R_{0}\right)\left(R_{25}-R_{0}\right)>\left(R_{12}-R_{0}\right)\left(R_{35}-R_{0}\right), \\
& \left(R_{14}-R_{0}\right)\left(R_{23}-R_{0}\right)>\left(R_{13}-R_{0}\right)\left(R_{24}-R_{0}\right)<\left(R_{12}-R_{0}\right)\left(R_{34}-R_{0}\right) .
\end{aligned}
$$

If $m_{4}$ is in the position shown in either Fig. 1a or Fig. 1b, then the first inequality sign in the second inequality and the second inequality sign in the third inequality must be reversed, while there is no change if $m_{4}$ is in the position shown in Fig. 1c.

Here, as in the convex case, we may select three of the five points of the pentagon arbitrarily, and we shall take as these three the points $m_{1}, m_{2}$, and $m_{3}$. Also let the order relation between $r_{12} ; r_{23}$, and $r_{13}$ be $r_{12}, r_{23} \leqq r_{0} \leqq r_{13}$.

In Fig. 4 draw $r_{12}$ with the masses $m_{1}$ and $m_{2}$ at its extremities. With these two points as centers draw semicircles with radius $r_{0}$ intersecting at $O$. With $O$ as center describe circular arcs $a_{1} b_{1}$ and $a_{2} b_{2}$, each with radius $r_{0}$. On account of the inequalities (17), $m_{3}$ will lie in the region $O b_{2} a_{2}, m_{4}$ in $O d_{1} d_{2}$, and $m_{5}$ in $O a_{1} b_{1}$, with the additional condition

$$
r_{12}, r_{23}, r_{34}, r_{14}, r_{24}, r_{15}, r_{25}, r_{45} \leqq r_{0} \leqq r_{13}, r_{25} .
$$

With the masses in these regions it may be shown that equations (14) are satisfied. Hence we have the companion theorem:

THEOREM 9.1. For every point $m_{3}$ in the region $\mathrm{Ob}_{2} a_{2}$, or on the boundary of this region, there is a single infinity of points $m_{4}$ in the region $O d_{1} d_{2}$ and a single infinity of points $m_{5}$ in the region $O a_{1} b_{1}$ which, together with the points $m_{1}$ and $m_{2}$, form an admissible concave pentagon. 
10. Symmetric pentagons. Suppose that

$$
r_{23}=r_{15}, \quad r_{14}=r_{24}, \quad r_{34}=r_{45}, \quad r_{13}=r_{25},
$$

and consequently that

$$
D_{23}=D_{15}, \quad D_{45}=D_{34}, \quad D_{14}=D_{24}, \quad D_{25}=D_{13} .
$$

The configurations Fig. 5 then present themselves in the form of isosceles trapezoids with vertices at the points $m_{1}, m_{2}, m_{3}$, and $m_{5}$ and with the point $m_{4}$ somewhere on the line perpendicular to and bisecting the parallel sides $r_{12}$ and $r_{35}$. The construction of the figure is the same as that of Fig. 2.

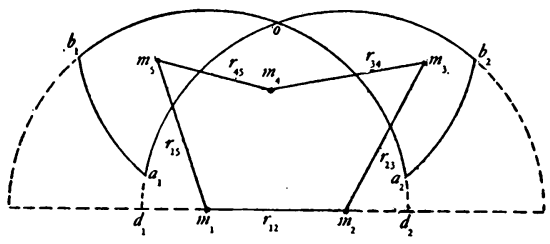

FIG. 4

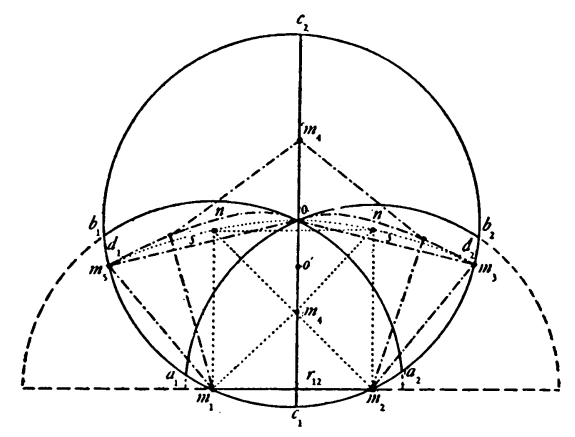

FIg. 5

Equations (14) reduce to the two independent equations

$$
\begin{aligned}
& \frac{\left(R_{24}-R_{0}\right)\left(R_{23}-R_{13}\right)}{\left(R_{13}-R_{0}\right)\left(R_{24}-R_{0}\right)-\left(R_{12}-R_{0}\right)\left(R_{34}-R_{0}\right)}=\frac{D_{12} D_{45}}{D_{24} D_{15}}, \\
& \frac{\left(R_{13}-R_{0}\right)\left(R_{34}-R_{0}\right)-\left(R_{24}-R_{0}\right)\left(R_{35}-R_{0}\right)}{\left(R_{24}-R_{0}\right)\left(R_{35}-R_{0}\right)-\left(R_{23}-R_{0}\right)\left(R_{34}-R_{0}\right)}=\frac{D_{15}}{D_{25}},
\end{aligned}
$$

and the mass equations (12) become

$$
\begin{aligned}
& \frac{m_{3}}{m_{1}}=\frac{m_{5}}{m_{2}}=\frac{D_{15}}{D_{12}} \frac{\left(R_{24}-R_{0}\right)\left(R_{13}-R_{23}\right)}{\left(R_{24}-R_{0}\right)\left(R_{35}-R_{0}\right)-\left(R_{13}-R_{0}\right)\left(R_{34}-R_{0}\right)}, \\
& \frac{m_{3}}{m_{2}}=\frac{m_{5}}{m_{1}}=\frac{D_{25}}{D_{12}} \frac{\left(R_{24}-R_{0}\right)\left(R_{13}-R_{23}\right)}{\left(R_{23}-R_{0}\right)\left(R_{34}-R_{0}\right)-\left(R_{24}-R_{0}\right)\left(R_{35}-R_{0}\right)}, \\
& \frac{m_{3}}{m_{4}}=\frac{m_{5}}{m_{4}}=\frac{D_{15}}{D_{24}} \frac{\left(R_{12}-R_{0}\right)\left(R_{34}-R_{0}\right)-\left(R_{13}-R_{0}\right)\left(R_{24}-R_{0}\right)}{\left(R_{13}-R_{0}\right)\left(R_{13}-R_{0}\right)-\left(R_{12}-R_{0}\right)\left(R_{35}-R_{0}\right)}, \\
& m_{5}=m_{3} .
\end{aligned}
$$

From either (19.1) or (19.2) it follows that we have also $m_{1}=m_{2}$. 
For convex pentagons all of the triangular area ratios in these equations are positive. For concave pentagons $D_{15} / D_{12}$ and $D_{25} / D_{12}$ are negative, if the point $m_{4}$ is above the diagonal $r_{13}$; otherwise they are positive. Moreover $D_{15} / D_{24}>0$.

The convex case. In Fig. 5, $r_{12}$ is regarded as fixed with the masses $m_{1}$ and $m_{2}$ at its extremities, while the point $m_{3}$ (and its symmetric point $m_{5}$ ) varies when the point $m_{4}$ moves from $O$ towards $c_{2}$, as $m_{4}$ must do since $r_{24} \geqq r_{0}$ for convex pentagons.

When the point $m_{4}$ is at $O$, equations (18.1) and (18.2) become, respectively,

$$
\begin{aligned}
\left(R_{12}-R_{0}\right)\left(R_{34}-R_{0}\right) D_{12} D_{45} & =0 \\
\left(R_{34}-R_{0}\right)\left[\left(R_{13}-R_{0}\right) D_{25}+\left(R_{23}-R_{0}\right) D_{15}\right] & =0 .
\end{aligned}
$$

In the first equation since neither $\left(R_{12}-R_{00}\right), D_{12}$, nor $D_{45}$ is zero, we must have $\left(R_{34}-R_{0}\right)=0$; whence $r_{34}=r_{0}$. It then follows that point $m_{3}$ is somewhere on the circular arc $m_{1} b_{1} c_{2} b_{2} a_{2} m_{2}$, and it can be proved that it is at some point $d_{2}$ between $a_{2}$ and $b_{2}$ with $m_{5}$ at the symmetric position $d_{1}$.

As the point $m_{4}$ moves from $O$ towards $c_{2}$, point $m_{3}$ moves on some curve $n$ from $d_{2}$ towards $O$, and in like manner, point $m_{5}$ moves from $d_{1}$ towards $O$ along some curve $n^{\prime}$ which is symmetric to $n$. When $m_{4}$ reaches a position on $O c_{2}$ such that the points $m_{3}$ and $m_{5}$ have moved in towards each other on curves $n$ and $n^{\prime}$, respectively, so that $r_{35}=r_{0}$, then the limit for permanent convex configurations is attained, since by inequalities (16), $r_{35} \geqq r_{0}$.

The concave case. As the point $m_{4}$ moves from $O$ towards $c_{1}$, as it must do for concave pentagons since $r_{24} \leqq r_{0}$, point $m_{3}$ moves on some curve $s$ from $d_{2}$ towards $O$, and in like manner, point $m_{5}$ moves on some curve $s^{\prime}$, symmetric to $s$ from $d_{1}$ towards $O$. However, since for concave pentagons $r_{35} \leqq r_{0}$, these curves are of no interest until points $m_{3}$ and $m_{5}$ reach positions on curves $s$ and $s^{\prime}$, respectively, where $r_{35}=r_{0}$.

Let the corresponding position of $m_{4}$ be $O^{\prime}$. If one starts with the point $m_{4}$ in this position, as it moves on towards $c_{1}$ points $m_{3}$ and $m_{5}$ move towards $O$. When they reach this position one has $r_{13}=r_{23}=r_{0}, D_{12}=0, D_{15} / D_{25}=-1$. Equations (18.1) and (18.2) are then both satisfied, regardless of the position of point $m_{4}$ on $O c_{1}$.

Thus, for a given $r_{12}$ and $r_{0}$, as point $m_{4}$ moves from $O$ towards $c_{2}$, point $m_{3}$ moves from $d_{2}$ towards $O$ along $n$, and point $m_{5}$ moves from $d_{1}$ towards $O$ along $n^{\prime}$, giving, in all positions for which $r_{35} \geqq r_{0}$, a convex pentagon; while as point $m_{4}$ moves from $O^{\prime}$ towards $c_{1}$, points $m_{3}$ and $m_{5}$ move towards $O$ along $s$ and $s^{\prime}$, respectively, giving, in all positions where $r_{35} \leqq r_{0}$, a concave pentagon. Since the mass ratios are all positive for all of these pentagons, as 
can be seen from equations (19.1), (19.2), and (19.3), we can state the following theorem:

THEOREM 10.1. For every $r_{12}$ and $r_{0}$ there exists a single infinity of permanent configurations with one mass at each of the vertices of an isosceles trapezoid and with the fifth on the line perpendicular to and bisecting the two parallel sides.

As the point $m_{4}$ moves from $O$ towards $c_{2}$, Fig. 5, and consequently, points $m_{3}$ and $m_{5}$ move from $d_{2}$ and $\dot{d}_{1}$ towards the symmetric points on $n$ and $n^{\prime}$ where $\left(r_{35}-r_{0}\right)$ vanishes, one sees from equations (19.1), (19.2), and (19.3) that the mass ratios

$$
\frac{m_{3}}{m_{1}}=\frac{m_{5}}{m_{2}}, \quad \frac{m_{3}}{m_{2}}=\frac{m_{5}}{m_{1}}, \quad \frac{m_{3}}{m_{4}}=\frac{m_{5}}{m_{4}},
$$

change steadily from the values

$$
\frac{D_{15}\left(R_{13}-R_{23}\right)}{D_{12}\left(R_{35}+R_{13}-2 R_{0}\right)}, \quad \frac{D_{25}\left(R_{13}-R_{23}\right)}{D_{12}\left(2 R_{0}-R_{23}-R_{35}\right)}, \quad 0
$$

respectively, to

$$
\begin{gathered}
\frac{D_{15}\left(R_{24}-R_{0}\right)\left(R_{13}-R_{23}\right)}{D_{12}\left(R_{0}-R_{13}\right)\left(R_{34}-R_{0}\right)}, \quad \frac{D_{25}\left(R_{24}-R_{0}\right)\left(R_{13}-R_{23}\right)}{D_{12}\left(R_{23}-R_{0}\right)\left(R_{34}-R_{0}\right)}, \\
\frac{D_{15}\left(R_{12}-R_{0}\right)\left(R_{34}-R_{0}\right)-\left(R_{13}-R_{0}\right)\left(R_{24}-R_{0}\right)}{D_{24}\left(R_{13}-R_{0}\right)^{2}}
\end{gathered}
$$

where the $D$ 's and $R$ 's are to be evaluated at the points in question. Similar limits on the mass ratio could be found for the concave case as $m_{4}$ moves from $O^{\prime}$ towards $c_{1}$. We therefore have the following theorem:

Theorem 10.2. For every $m_{1}=m_{2}>0, m_{3}=m_{5}>0$, and $m_{4}>0$, there is one and only one isosceles trapezoid configuration.

11. The case of three points on a line. Let $m_{4}$ be on the line $r_{13}$ joining $m_{1}$ and $m_{3}$, and let $m_{2}$ and $m_{5}$ be equidistant from $r_{13}$. We have at once

$$
\begin{aligned}
& r_{12}=r_{15}, \quad r_{23}=r_{35}, \quad r_{24}=r_{45}, \\
& D_{25}=0, \quad D_{35}=D_{23}, \quad D_{45}=D_{24}, \quad D_{15}=-D_{12},
\end{aligned}
$$

and the equations (14) reduce to the single equation

$$
\frac{\left(R_{14}-R_{0}\right)\left(R_{23}-R_{0}\right)-\left(R_{13}-R_{0}\right)\left(R_{24}-R_{0}\right)}{\left(R_{13}-R_{0}\right)\left(R_{24}-R_{0}\right)-\left(R_{12}-R_{0}\right)\left(R_{34}-R_{0}\right)}=\frac{-D_{34} D_{15}}{D_{14} D_{35}} .
$$

Let points $m_{1}$ and $m_{3}$ be fixed. Then for a given $m_{2}$ and for any position of point $m_{4}$, the equation (21) to be satisfied, by the coordinates of point $m_{5}$, 
is the equation of a straight line. But for a solution the coordinates of $m_{5}$ must also satisfy the first two equations of (20). (The remaining equations of (20) are then automatically satisfied.) These equations, when rationalized, represent circles with centers at the points $m_{1}$ and $m_{3}$ and with radii $r_{12}$ and $r_{23}$, respectively.

In Fig. 6 draw $r_{13}$ with $m_{1}$ and $m_{3}$ at its extremities. Take point $m_{2}$ anywhere not on this line, and draw the circles of the above equations. It is obvious that these two equations are satisfied by real points if, and only if, point $m_{5}$ lies at the intersection of their circles. That is, point $m_{5}$ will either be at the point $a$, Fig. 6 , or it will coincide with point $m_{2}$. Thus it may be in one of two positions in so far as these two equations are concerned.

Equation (21) must be satisfied also. By Theorem 4.1, the left member of this equation must be either 0 or -1 . If it is 0 , the equation reduces to

$$
D_{34}=0 \text {, }
$$

which is the equation of a straight line passing through points $m_{1}$ and $m_{2}$; therefore, in order for the point $m_{5}$ to satisfy all the equations, it must coincide with $m_{2}$. This is a case of no interest as $r_{25}$ may vanish for neither convex nor concave pentagons.

Suppose the left-hand member has the value -1 . This gives the equation

$$
\frac{R_{12}-R_{0}}{R_{23}-R_{0}}=\frac{R_{14}-R_{0}}{R_{34}-R_{0}} .
$$

The question arises as to whether or not the line with this equation can be made to pass through the point $a$. In other words, are there configurations other than the one where points $m_{2}$ and $m_{5}$ coincide? The proof that the answer to this question is in the affirmative is not difficult, but an illustrative example would, perhaps, be more instructive.

Let the point $m_{4}$ be midway between points $m_{1}$ and $m_{3}$. The equation (22) is always satisfied, and if the point $m_{2}$ is given a position such that the mass ratios are all positive, we have a permanent configuration which, in general, will be a rhombus with vertices at $m_{1}, m_{2}, m_{3}$, and $m_{5}$, and with $m_{4}$ at its center.

In Fig. 7, draw $r_{13}$, and consider the point $m_{2}$ as it moves on a line perpendicular to $r_{13}$ at its mid-point $m_{4}$. The positions that it may assume are limited only by the regions for positive masses, since the condition (22) is satisfied for any position of $m_{2}$ regardless of what $r_{0}$ may be. Moreover whereever point $m_{2}$ may be on this line, we have, from (12),

$$
\frac{m_{1}}{m_{3}}=1, \quad \frac{m_{2}}{m_{5}}=1 \text {. }
$$




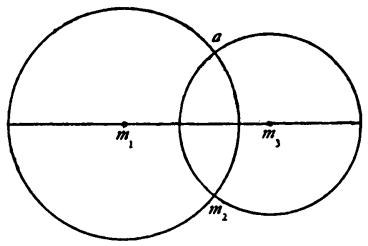

Fig. 6

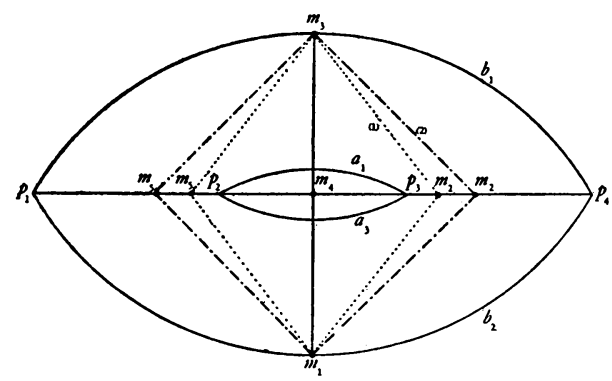

Fig. 7

With points $m_{1}$ and $m_{3}$ as centers draw circular arcs $a_{1}$ and $a_{3}$ with a radius $r_{0},\left(r_{0}<r_{13}\right)$, such that the distance between their intersections $p_{2}$ and $p_{3}$ is equal to $r_{0}$. Since $r_{25} \geqq r_{0}$, we have, with point $m_{2}$ at $p_{3}$ and $m_{5}$ at $p_{2}$, the rhombus configuration in which the point $m_{2}$ is as close to $m_{5}$ as possible for a given $r_{13}$. In this case the mass equations (12) show that

$$
\frac{m_{3}}{m_{2}}=0, \frac{m_{4}}{m_{3}}=\frac{2\left(R_{0}-R_{13}\right)}{\left(R_{14}-R_{0}\right)}>0, \frac{m_{3}}{m_{5}}=0, \frac{m_{1}}{m_{5}}=0, \frac{m_{4}}{m_{5}}=0 .
$$

The greatest possible distance between the points $m_{2}$ and $m_{5}$, for a given $r_{13}$, is obtained when we choose an $r_{0}=r_{13}$. Draw circular arcs $b_{1}$ and $b_{2}$ with such a radius. Then, with $m_{2}$ and $p_{4}$ and $m_{5}$ at $p_{1}$, we have the rhombus configuration in which the distance between points $m_{2}$ and $m_{5}$ is as great as possible. Here, we have

$$
\frac{m_{3}}{m_{2}}=\infty, \quad \frac{m_{4}}{m_{3}}=0, \quad \frac{m_{3}}{m_{5}}=\infty, \quad \frac{m_{1}}{m_{5}}=\infty, \quad \frac{m_{4}}{m_{5}}=\frac{2\left(R_{0}-R_{25}\right)}{\left(R_{24}-R_{0}\right)}>0 .
$$

THEOREM 11.1. Given $r_{13}$ with masses $m_{1}$ and $m_{3}$ at its extremities and with the mass $m_{4}$ at its center, for a properly chosen $r_{0}$, and for all positions of $m_{2}$ and $m_{5}$ on a line perpendicular to $r_{13}$ at $m_{4}$ such that the mass ratios have values lying between those given in (23) and those in (24), the configuration is permanent and is a rhombus with vertices at $m_{1}, m_{2}, m_{3}$, and $m_{5}$, and with $m_{4}$ at its center.

In concluding this section we may add that if any four of the masses are on the same straight line, the fifth mass will be on this line also. This follows readily from equations (14).

12. The triangle with two interior masses. One other possibility of a concave pentagon is that of a triangle with one mass at each vertex and with the two remaining masses in its interior. That no such configuration, however, can form an admissible pentagon follows from the inequalities

$$
r_{12}, r_{23}, r_{34}, r_{14}, r_{24}, r_{15}, r_{35}, r_{45} \leqq r_{0} \leqq r_{13}, r_{25}
$$


(see $\$ 9$ ). To construct a pentagon of this type one must alter the above order relation that must exist among the sides and diagonals, and the result is always to cause some of the masses to be negative.

13. Numerical solutions. We consider the following examples:

EXAMPLE 1. An interesting example of a convex permanent configuration is that of the regular pentagon. In Fig. 5 as the point $m_{4}$ moves upward from $O$ towards $c_{2}$ and the points $m_{3}$ and $m_{5}$ move towards each other along curves $n$ and $n^{\prime}$, somewhere along these paths the configuration becomes that of a regular pentagon. When this position is reached, the equations (18.1) and (18.2) reduce to the single equation

$$
\frac{R_{24}-R_{0}}{2 R_{0}-R_{12}-R_{24}}=\frac{D_{15}}{D_{25}}=\frac{r_{12}}{r_{24}}
$$

or

$$
\left(r_{0}^{3}-r_{24}^{3}\right) r_{12}^{2} r_{24}-2 r_{12}^{3} r_{24}^{3}+r_{0}^{3}\left(r_{24}^{3}+r_{12}^{3}\right)=0 .
$$

For any given value of $r_{13}, r_{24}$ may be computed from the obvious relation

$$
r_{24}=1.6180 r_{12},
$$

and $r_{0}$ may be found from (26). It follows readily that

$$
\begin{aligned}
& \frac{r_{0}}{r_{12}}=1.3076, \quad \frac{r_{23}}{r_{12}}=\frac{r_{34}}{r_{12}}=\frac{r_{45}}{r_{12}}=\frac{r_{15}}{r_{12}}=1, \\
& \frac{r_{13}}{r_{12}}=\frac{r_{24}}{r_{12}}=\frac{r_{14}}{r_{12}}=\frac{r_{25}}{r_{12}}=\frac{r_{35}}{r_{12}}=1.6180,
\end{aligned}
$$

and from (19.1), (19.2), (19.3), together with (25), that $m_{1}=m_{2}=m_{3}=m_{4}=m_{5}$.

EXample 2. Perhaps the most simple concave permanent configuration in the five-body problem is that of a square with one mass at each of its vertices and with the fifth mass at its center. In Fig. 5 this particular configuration is passed through as the point $m_{4}$ moves from $O$ towards $c_{1}$ and as points $m_{3}$ and $m_{5}$ move towards each other along curves $s$ and $s^{\prime}$. Equations (18.1) and (18.2) are now identically satisfied for any value of $r_{0}$, while the mass equation (19.1) shows that

$$
\frac{m_{3}}{m_{1}}=\frac{m_{5}}{m_{2}}=1
$$

and since for all of the symmetric configurations, Fig. 5, $m_{3}=m_{5}, m_{1}=m_{2}$, we have $m_{1}=m_{2}=m_{3}=m_{5}$. 
The equation (19.3) becomes

$$
\begin{aligned}
\frac{m_{3}}{m_{4}}=\frac{m_{5}}{m_{4}}=\frac{1}{2} \frac{R_{34}-R_{0}}{2 R_{0}-R_{12}-R_{13}} & =\frac{\left(r_{0}^{3}-r_{34}^{3}\right)_{12}^{3} r_{13}^{3}}{2 r_{34}^{3}\left(2 r_{12}^{3} r_{13}^{3}-r_{0}^{3} r_{13}^{3}-r_{0}^{3} r_{12}^{3}\right)} \\
& =\frac{\left(8 r_{0}^{3}-2(2)^{1 / 2} r_{12}^{3}\right)}{2\left[4(2)^{1 / 2} r_{12}^{3}-r_{0}^{3}\left(1+2(2)^{1 / 2}\right)\right]},
\end{aligned}
$$

since $r_{13}^{2}=2 r_{12}^{2}=4 r_{34}^{2}$.

Thus, while equations (18.1) and (18.2) are satisfied for any $r_{0}$, the mass ratios $m_{3} / m_{4}=m_{5} / m_{4}$ are not necessarily positive for any value of $r_{0}$. It is found that for the last right-hand member of (27) to be positive we must have

It then follows that

$$
0.878<\frac{r_{12}}{r_{0}} \leqq 1.000
$$

$$
0.439<\frac{m_{3}}{m_{4}}=\frac{m_{5}}{m_{4}} \leqq 1.414 .
$$

This result shows that the central mass is arbitrary within certain limits. In general, if a permanent configuration is given, the masses are uniquely determined.

EXAMPLE 3. In Fig. 7 consider the rhombus configuration (1) with masses $m_{1}, m_{2}, m_{3}$, and $m_{5}$ at its vertices and with the mass $m_{4}$ at its center. By Theorem 11.1, for a given $r_{0}$ the point $m_{2}$ may be anywhere on the line perpendicular to $r_{13}$ at its mid-point such that the mass ratios are positive. It is readily found that

$$
\begin{array}{ll}
\frac{r_{0}}{r_{12}}=1.042, \quad \frac{r_{23}}{r_{12}}=\frac{r_{35}}{r_{12}}=\frac{r_{15}}{r_{12}}=1, & \frac{r_{14}}{r_{12}}=\frac{r_{34}}{r_{12}}=0.781, \\
\frac{r_{24}}{r_{12}}=\frac{r_{45}}{r_{12}}=0.625, \quad \frac{r_{25}}{r_{12}}=1.249, & \frac{r_{13}}{r_{12}}=1.562 ;
\end{array}
$$

and from the mass equations (12) we have,

$$
\frac{m_{1}}{m_{3}}=1, \frac{m_{2}}{m_{3}}=\frac{m_{5}}{m_{3}}=\frac{m_{5}}{m_{1}}=2.617, \frac{m_{4}}{m_{3}}=0.536, \frac{m_{5}}{m_{4}}=4.878, \frac{m_{2}}{m_{5}}=1 .
$$

As the point $m_{2}$ moves on towards $p_{4}$ and the point $m_{5}$ moves towards $p_{1}$, Fig. 7, the polygon moves through the square configuration (2), but this has already been discussed in Example 2 in connection with Fig. 5.

The University of Chicago,

Chicago, Ill. 